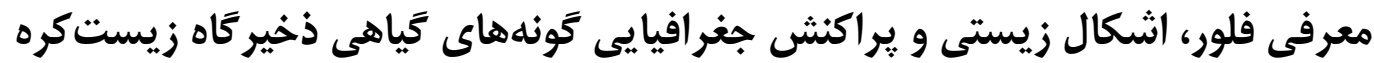

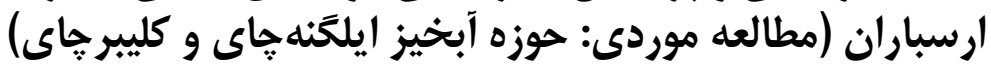

\author{
الله وردى محمدز اده'، رضا بصيرى ؛ مهررانكَيز يولاديانَّ و نسيم زينلى
}

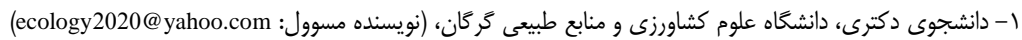

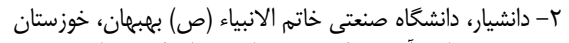

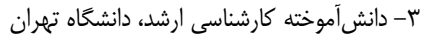

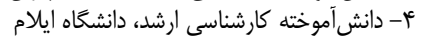

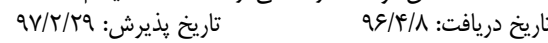

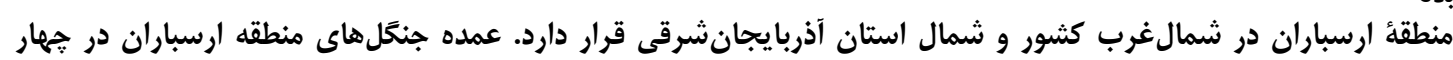

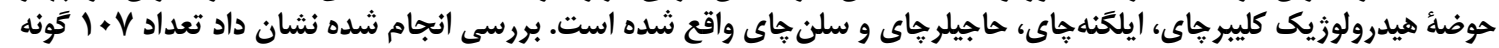

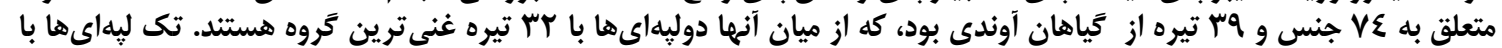

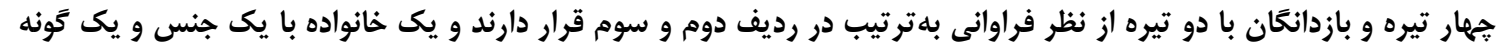

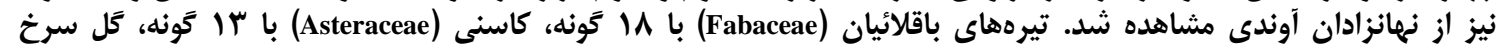
(Rosaceae)

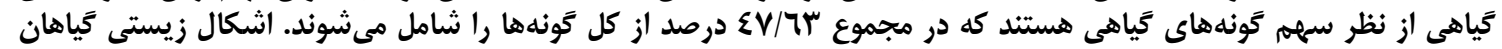

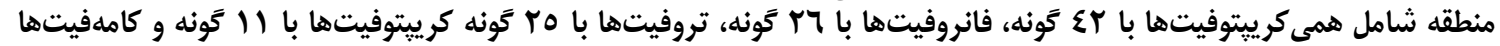

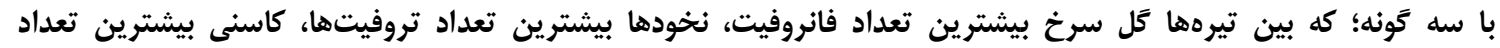

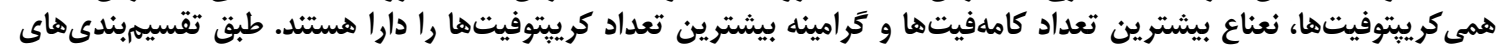

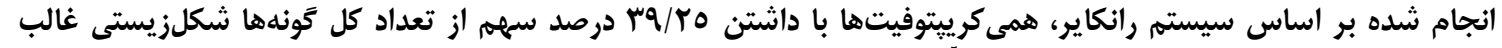

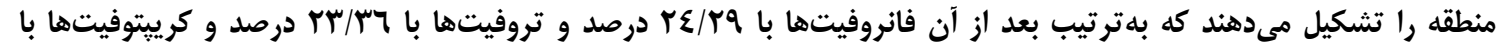

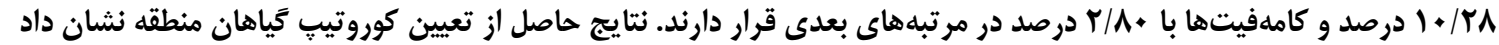

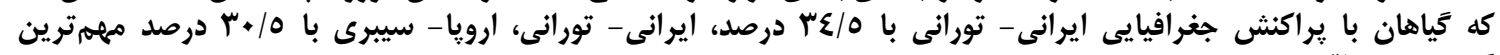
كرووهاى منطقه هستند.

وازههاى كليدى: يوشش تياهى، شكل زيستى، يراكنش جغرافيايى، طبقهبندى رانكاير، منطقه ارسباران

فعاليتهايى است كه بهمنظور سازماندهى و ثبت تنوع كياهان

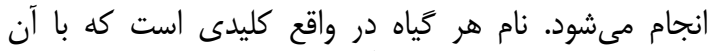

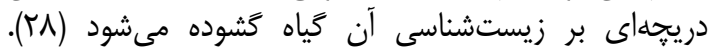

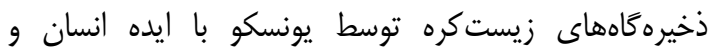

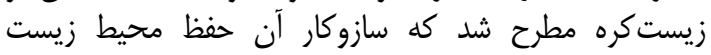

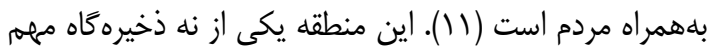

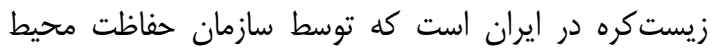

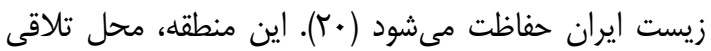

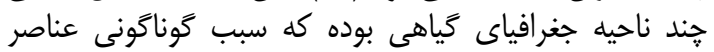

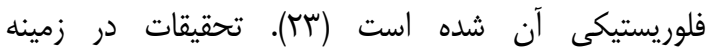

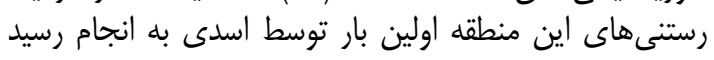
(V)

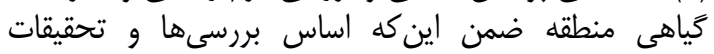

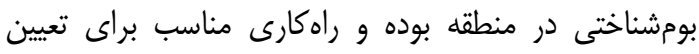

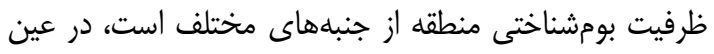

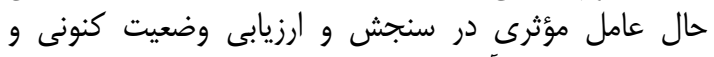

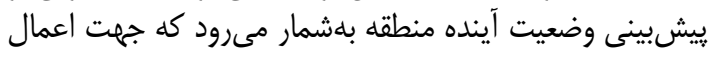

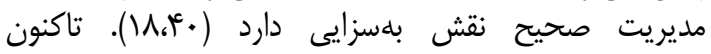

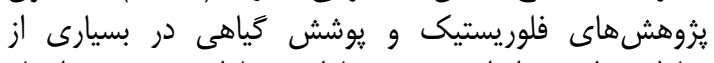

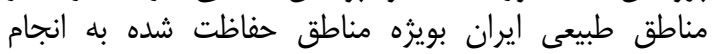
رسيده است. حمزه و همكاران (ساب) در تجزيه و و تحليل
مقدمه

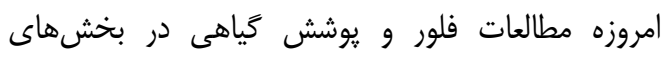

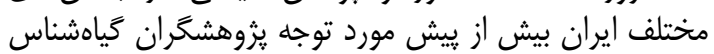

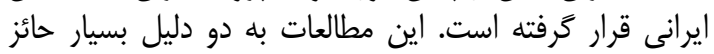

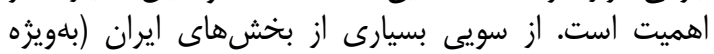

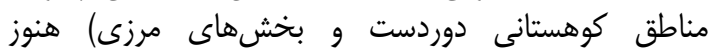

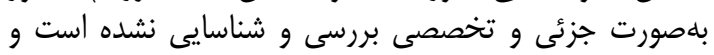

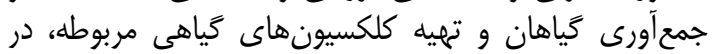

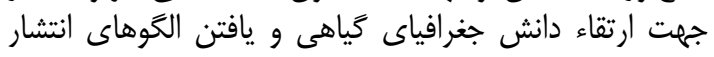

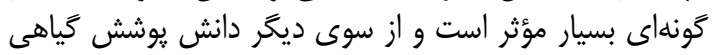

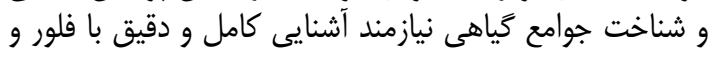

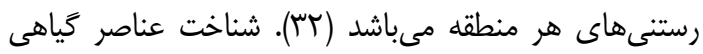

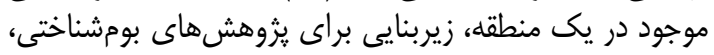

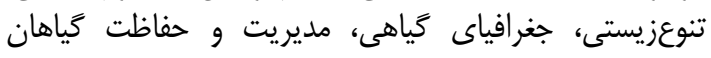

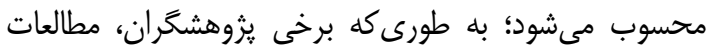

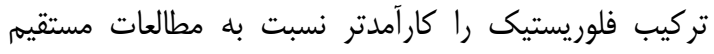

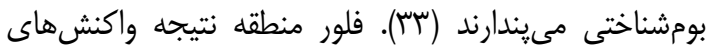

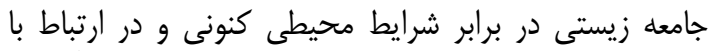

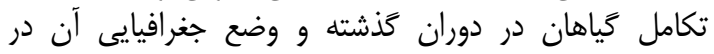

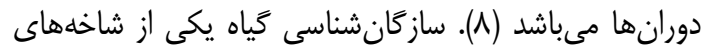

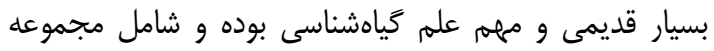


س

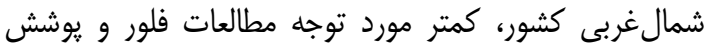

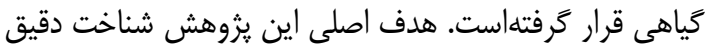

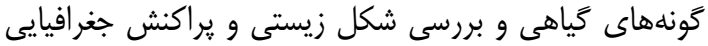

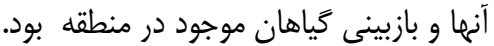

مواد و روشها

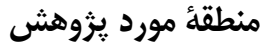

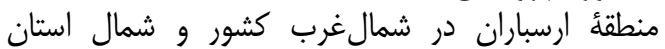

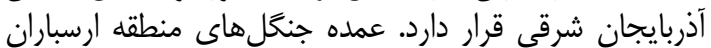

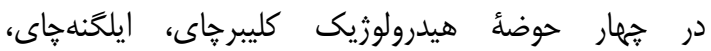

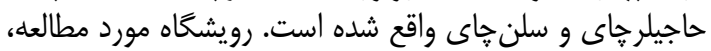

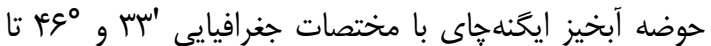

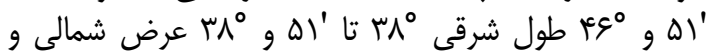

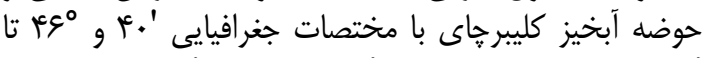

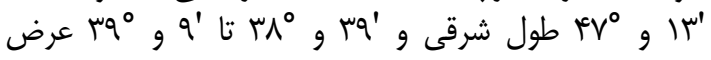

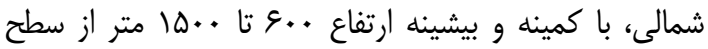

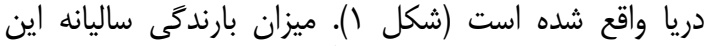

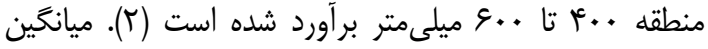

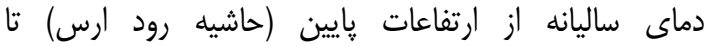

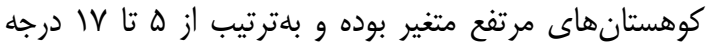
سانتى

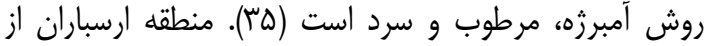

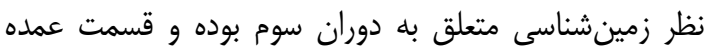

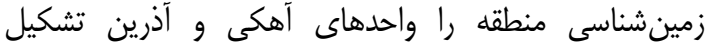

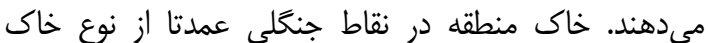

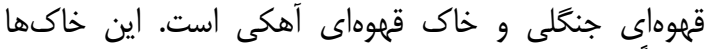

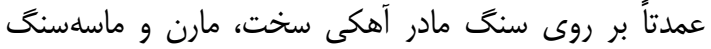

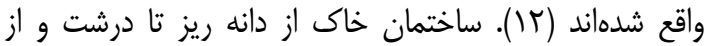

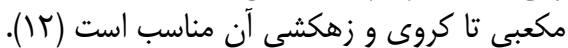

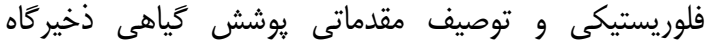

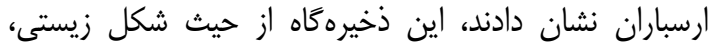
همى كرييتوفيتها

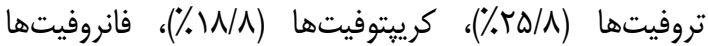

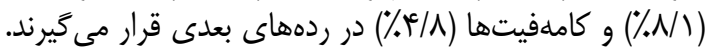

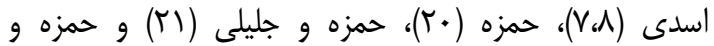

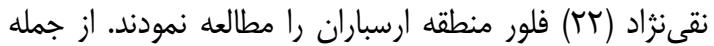

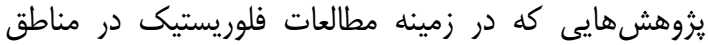

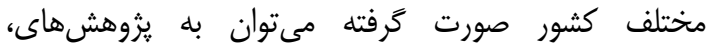

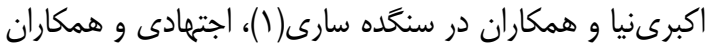

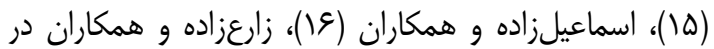

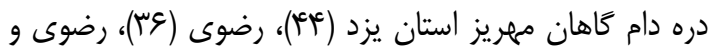

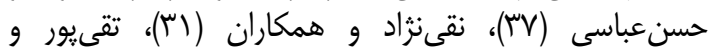

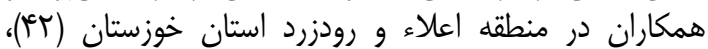

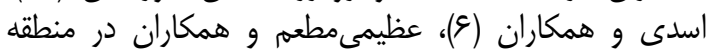

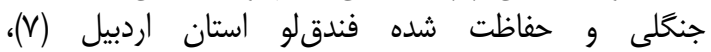

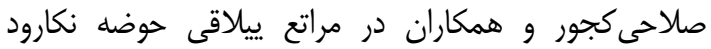

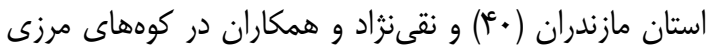

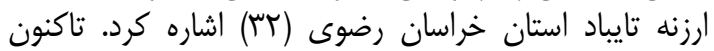

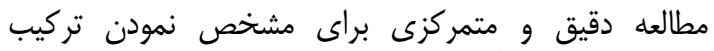

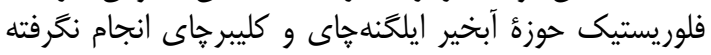

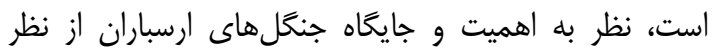

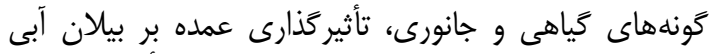

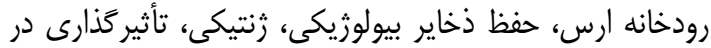

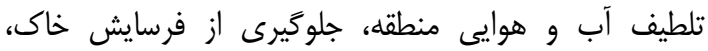

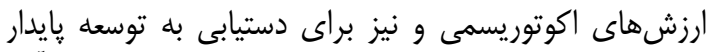

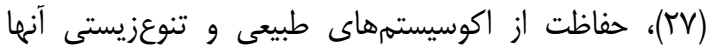

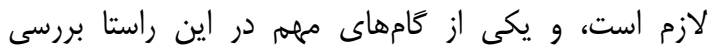

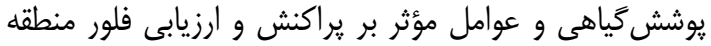
مورد نظر در اين جنگل هاست (YV) دليل كوهستانى بودن و قرار كرفتن

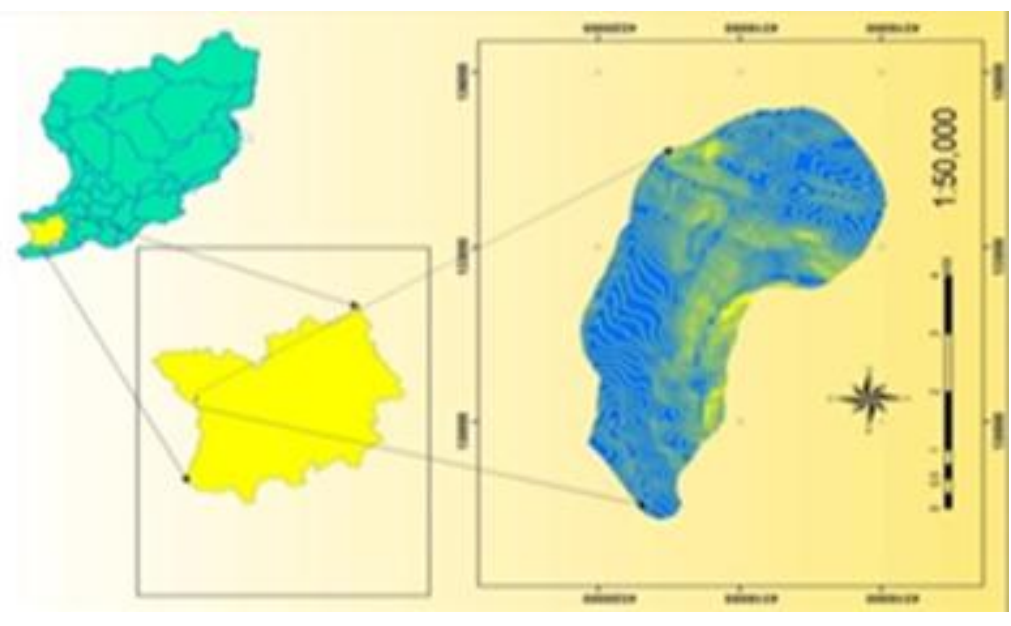

شكل ا- موقعيت محدوده مورد يثوهش در كشور و در استان آذربايجان شرقى

Figure 1. Location of research area in the country and East Azarbaijan province 
(IV)

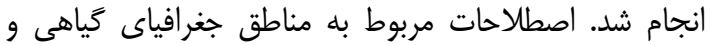

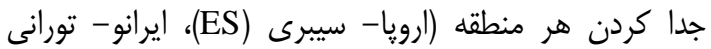
(IT)

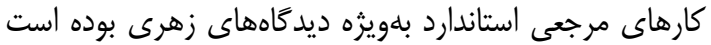

\section{نتايج و بحث}

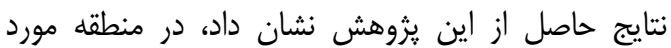

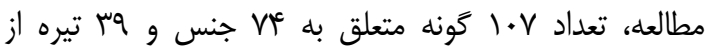

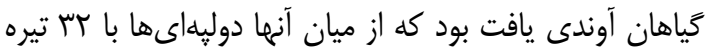

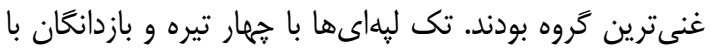

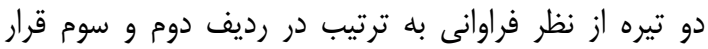

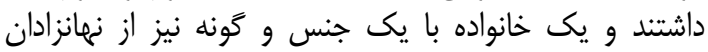

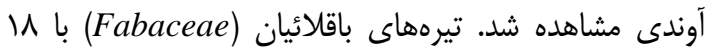

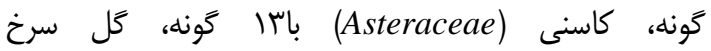
(Rosaceae)

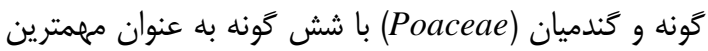

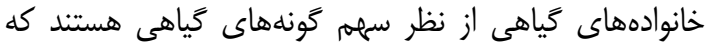

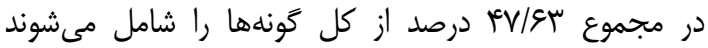

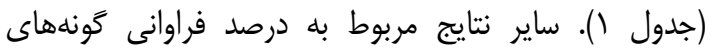

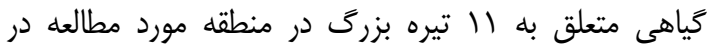
شكل (T) نشان داده شده است.

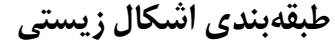

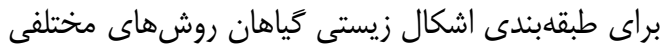

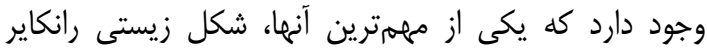

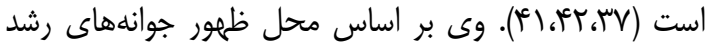

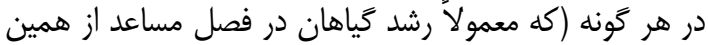

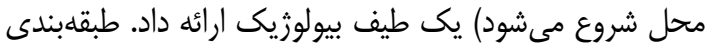

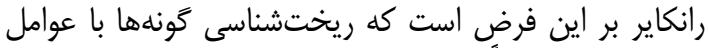

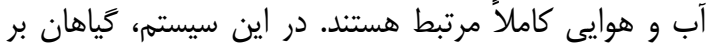

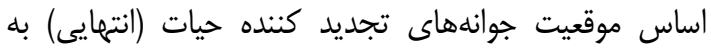
ينجدسته فانروفيتها(Phanerophytes)، (Chameophyte)، همى كريتوفيتوفيتها (Hemicryptophyt)، كرييتوفيتها (Cryptophytes) و تروفيتها (Therophyte)

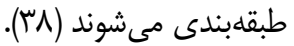
روش كار

ابتدا بهمنظور آشنايى با منطقه، نقشههاى جغرار افيايى ناحيه

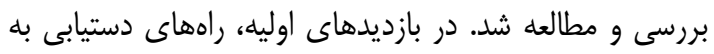

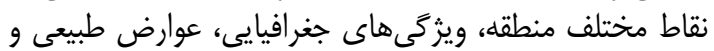

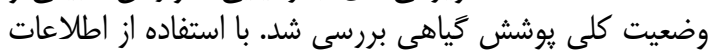

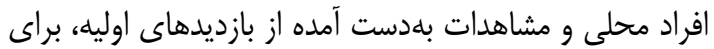

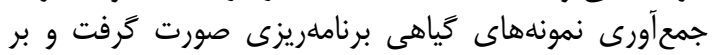

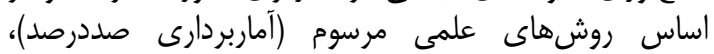

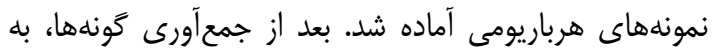

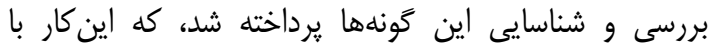

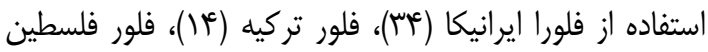

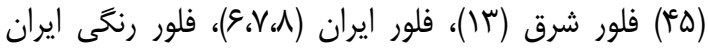

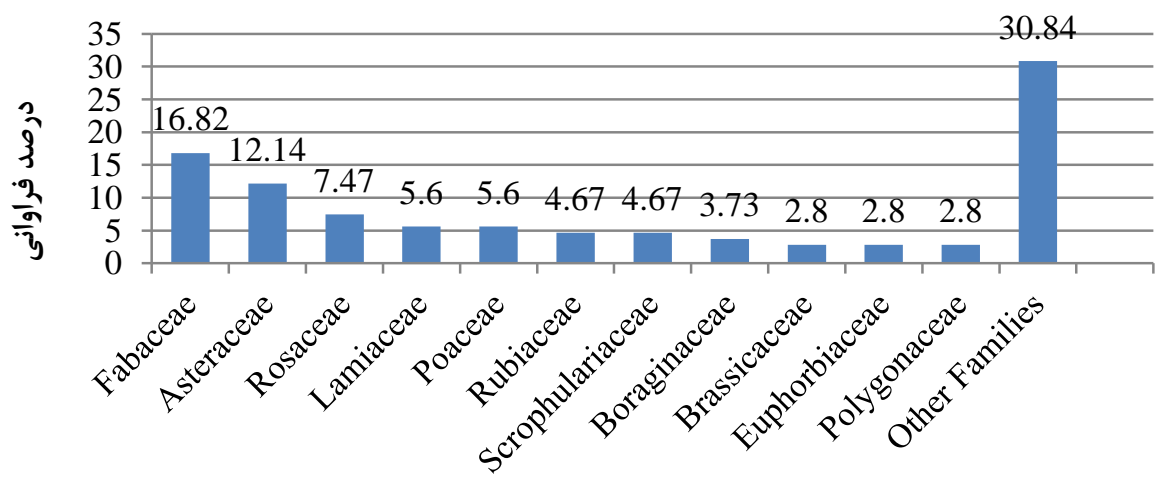

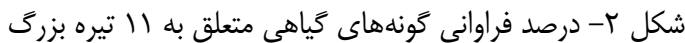

Figure 2. Percentage of plant species belonging to 11 large dams

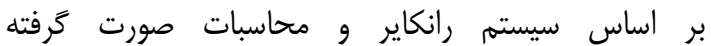

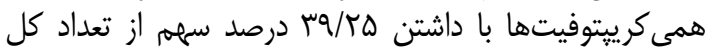

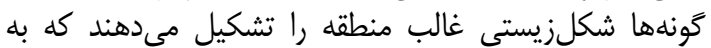

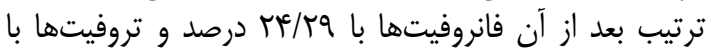

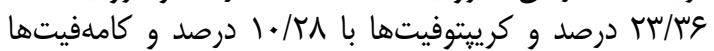

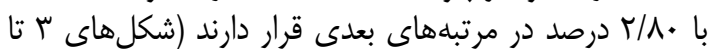

اشكال زيستى گياهان منطقه شامل همى كرييتوفيتها با بان

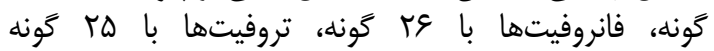

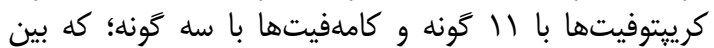

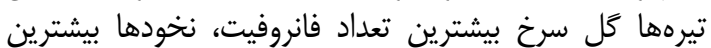

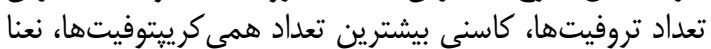

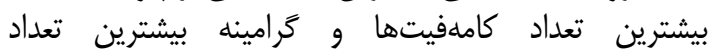

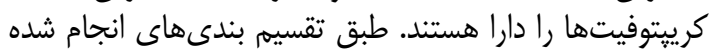




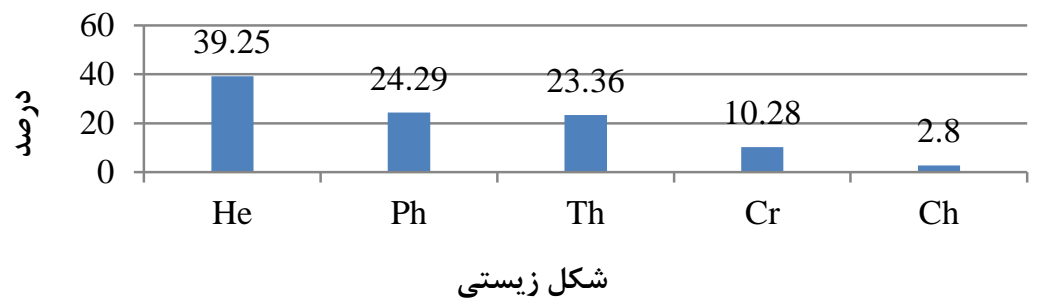

شكل س- درصد فراوانى شكلهاى زيستى گونههاى گياهى منطقه ch: كامفيتها، Cr: كرييتوفيتها، Ph: فانروفيتها، He: همى كرييتوفيتها، Th

Figure 3. Percentage of the abundance of bioforms of plant species in region, Ch: Camphites, Cr: Cryptophytes, $\mathrm{Ph}$ : Phanophytes, He: Hemicryptophytes, Th: Trophites

مهرمترين گروه منطقه است. ساير نتايج مربوط بله يراكنش

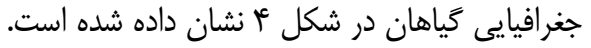

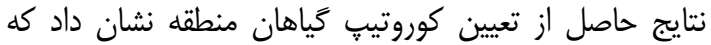

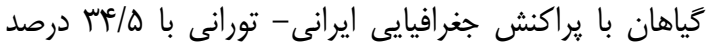

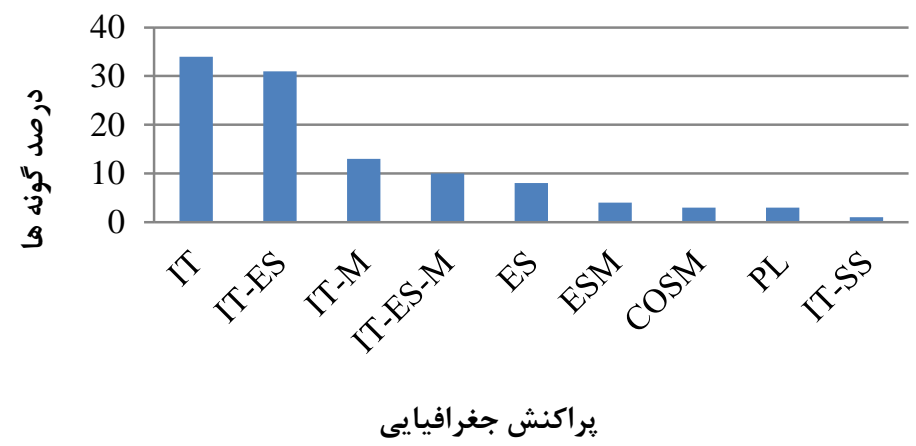

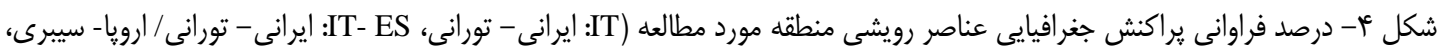

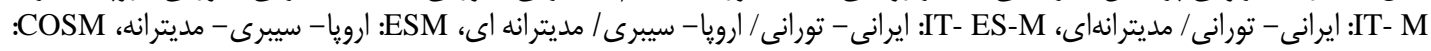

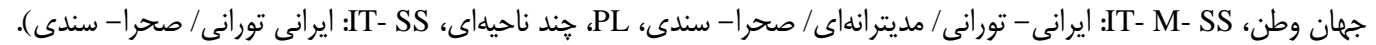
Figure 4. Percentage of geographic distribution of vegetative elements in the study area (IT: Iran-Turanian, IT-ES: Iran-Turanian/ Europe-Siberian, IT-M: Iran-Turanian/ Mediterranean, IT-ES-M: Iranian- Turanian/ EuropeanSiberian/ Mediterranean, ESM: Europe- Siberia- Mediterranean, COSM: World, IT-M-SS: Iran- Turanian/ Mediterranean/ Sahara- Sindi, PL, Multi- Region, IT- SS: Iranian Turani/ Sahra- Sandy)

خانوادها از نظر تعداد گونهاء Fabaceae Rosaceae Poaceae

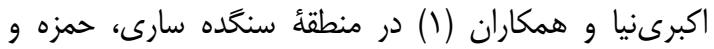

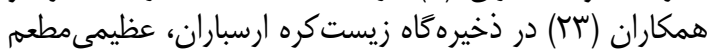

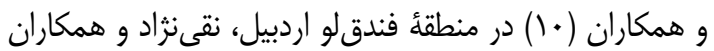

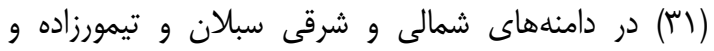

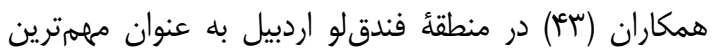

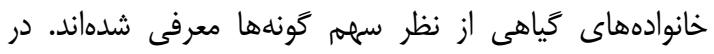

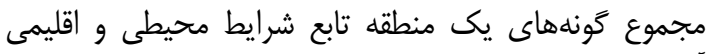

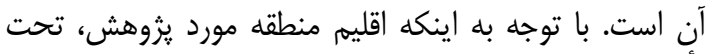

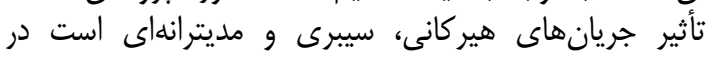

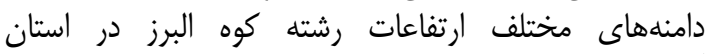

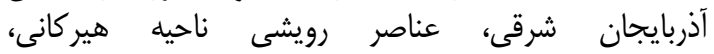

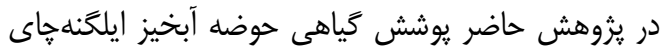

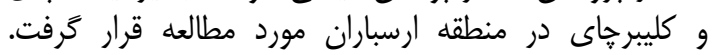

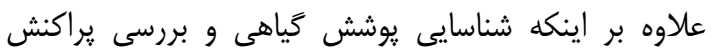

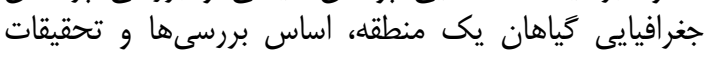

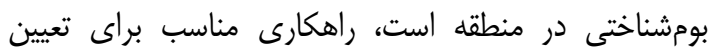

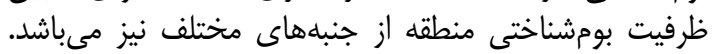

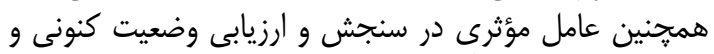

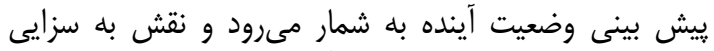

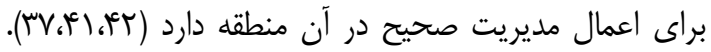

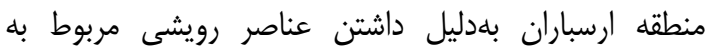

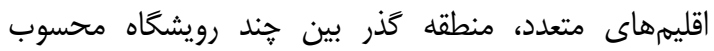

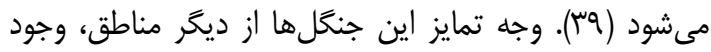

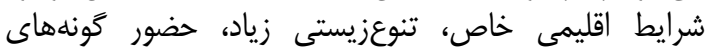

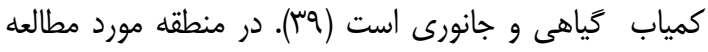

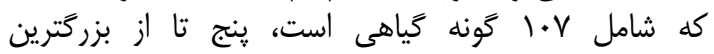


تروفيتها (يكسالهها) در اين يزوهش (حدود بآ درصد) نشان

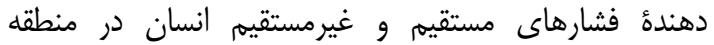

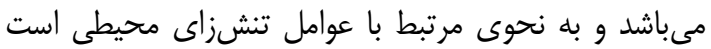

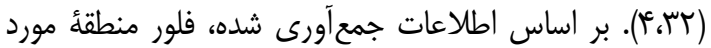

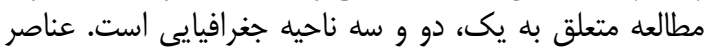

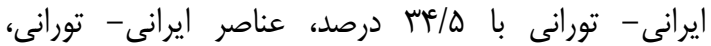

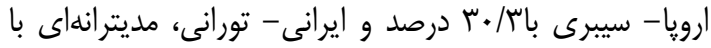

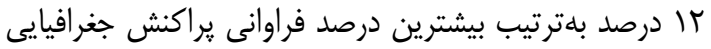

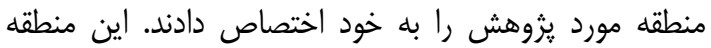

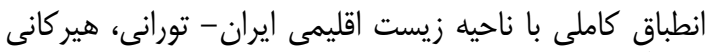

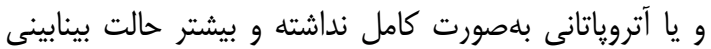

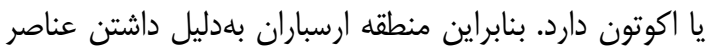

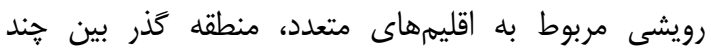

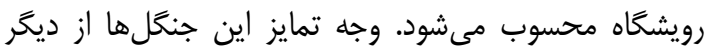

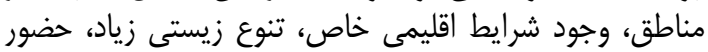

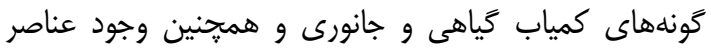

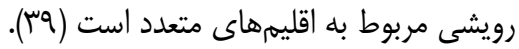

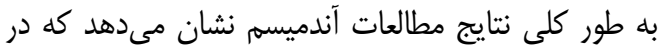

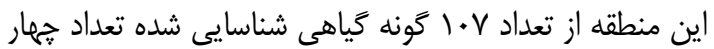

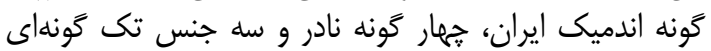

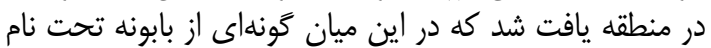
(Anthemis tinctoria L.)

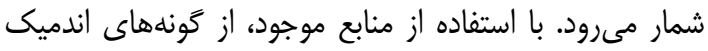

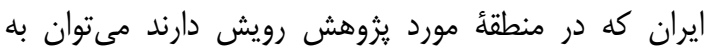
Delphinum CCentaurea aziziana Rech. f., Verbascum g ochroleucum Stev. Ex Dc. szovitsianum Boiss.

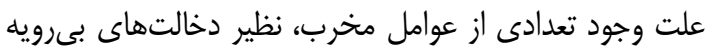

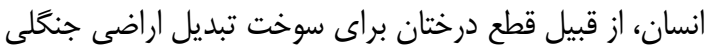

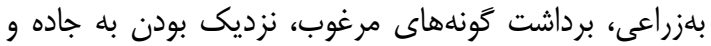

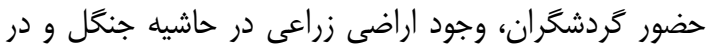

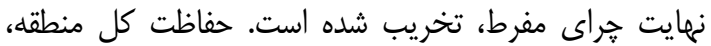

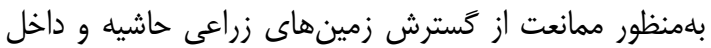

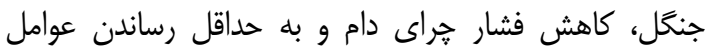

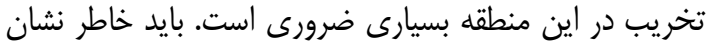

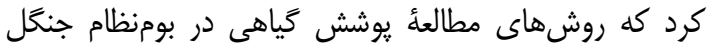

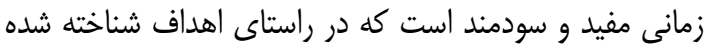
مديريت جنغل قرار كيرند.

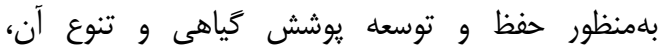

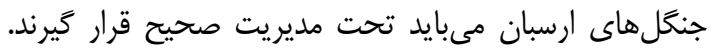

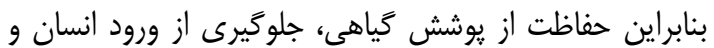

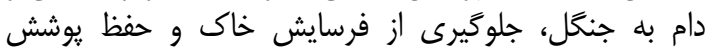

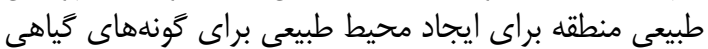
و جانورى در منطقه لازم است.

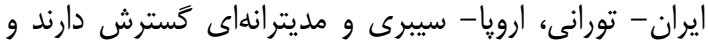

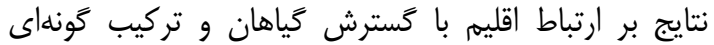

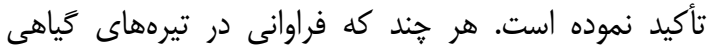
Fosaceae Poaceae ، Fabaceae اميدوار كننده است، اما حضور نسبى تيره Psteraceae نكران

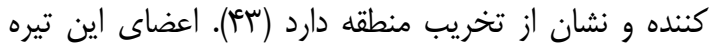

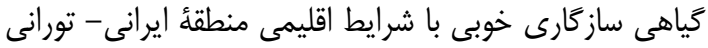

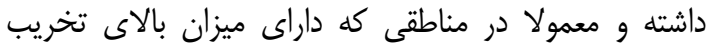

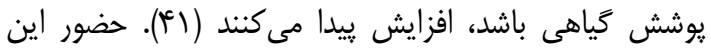

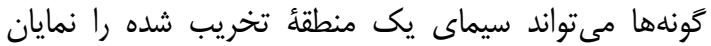

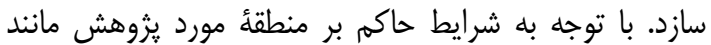

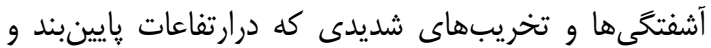

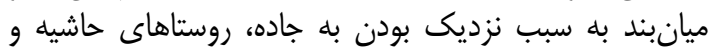

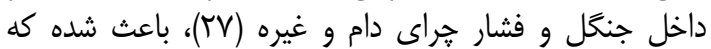
بسيارى از كونههاى تيره

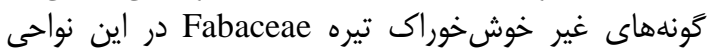

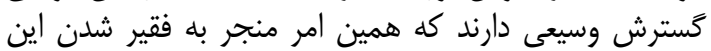

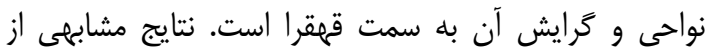

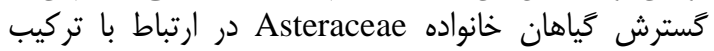

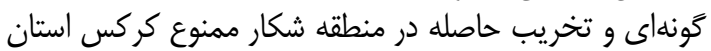

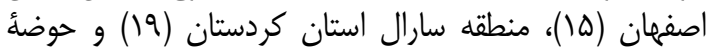

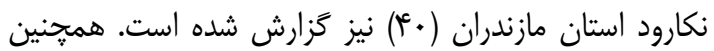
وجود كَونهايى مانند: Paliurus spina-christi Miller Mespilus ؛Crataegus orientalis Pall. ex M.B. 9 Urticadioica CCirsium arvense !germanica Bromus sterilis L.

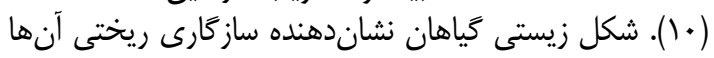

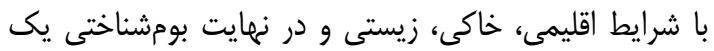

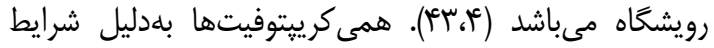

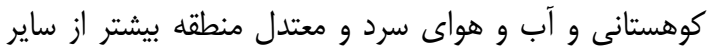

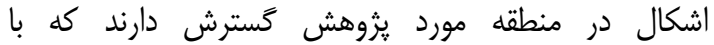

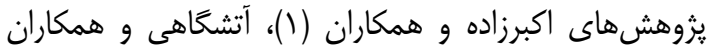

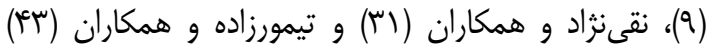

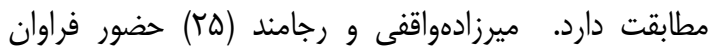

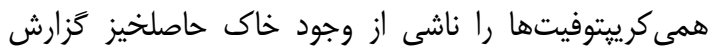

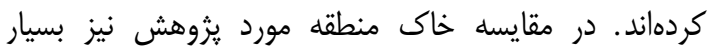

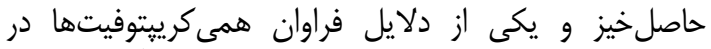

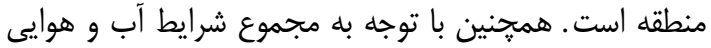

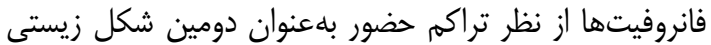

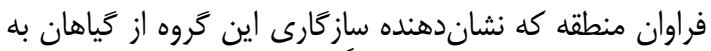

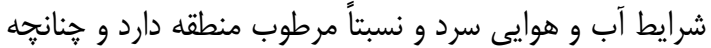

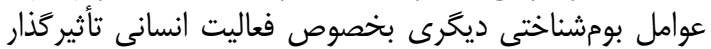

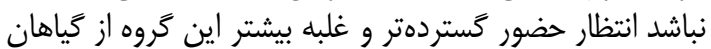

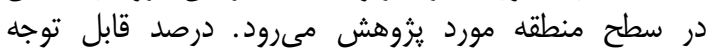




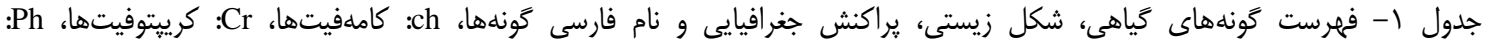

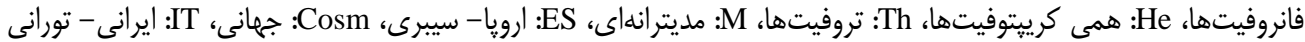

Table 1. List of plant species, biological form, geographical distribution and Persian names of species, ch: Camphites, Cr: cryptophytes, Ph: Phanrophytes, He: Hemic Cryptophytes, Th: Trophites, M: Mediterranean, Es: Europe-Siberian, Cosm: World, IT: Iranian-Turanian

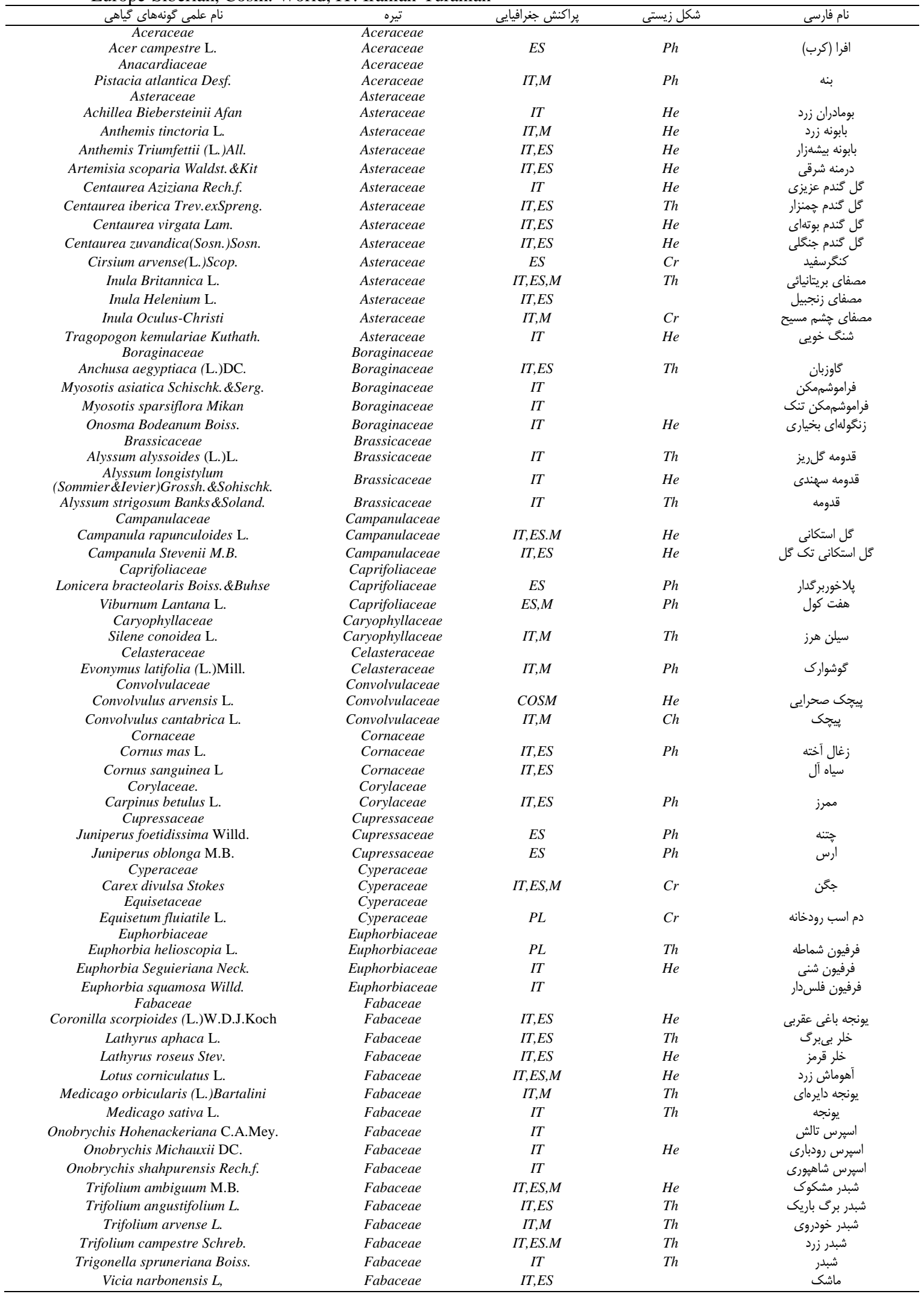




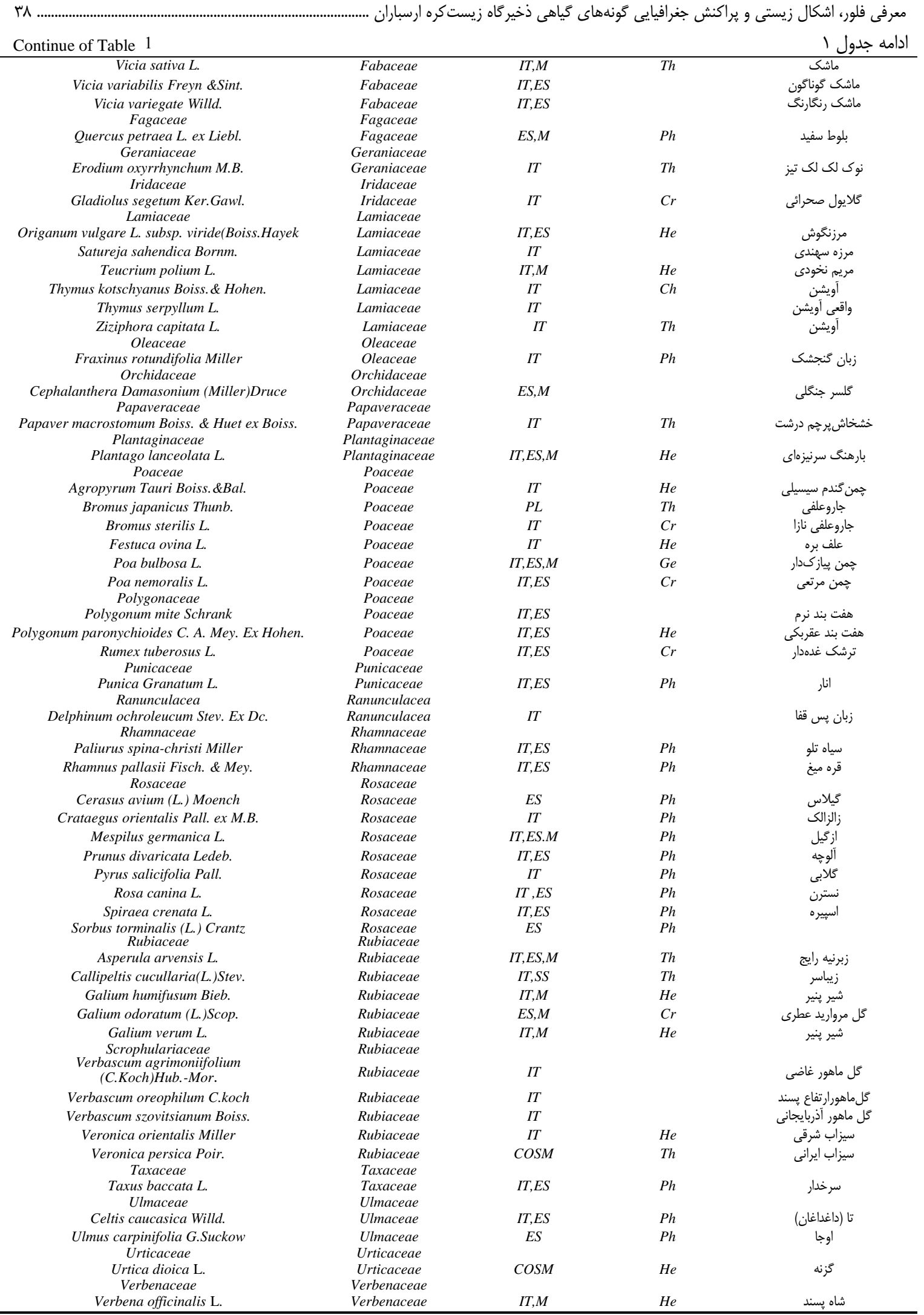


1. Akbarzadeh, M. 2006. Study on floristic, life form and chorology plants rangelands of Vaz. Journal of Pajouhesh and Sazandegi, 20(2): 198-199 (In Persian).

2. Alijanpour, A., J. Eshaghi Rad and A. Banej Shafiei. 2011. The effect of physiographical factors on qualitative and quantitative characteristics of Cornus mas L. in Arasbaran forests. Iranian Journal of Forest and Poplar Research, 19(3): 396-407. (In Persian)

3. Alijanpour, A. 2000. The investigation and determination of optimum inventory and its application in Arasbaran forests, $\mathrm{PhD}$ thesis, Tehran University, 160pp. (In Persian)

4. Archibold, O.W. 1995. Ecology of world vegetation. Chapman and Hall Inc, London, 509p.

5. Assadi, M., A. Masomi, M. Khatamsaz and V.A. Mozafarian. 1988-2011. Flora of Iran, Research Institute of Forests and Rangelands Publication, Tehran, 1-71 (In Persian).

6. Atashgahi, Z., H. Ejtehadi and H. Zare. 2009. Study of floristics, life form and chorology of plants in the east of Dodangeh forests, Mazandaran province, Iran. Journal of Biology, 22(2): 193-203. (In Persian)

7. Azimie Motam, F., R. Talaii, F. Asiabizade and M. Hoshyar. 2011. Flora, life form and chorology of plants species of the region forest and protect (Ardabil Province). Taxonomy and Biosystematics, 3(9): 75-88 (In Persian).

8. Asri, Y., M. Rabei, B. Hamze and A. Jalili. 2009. Flora of Artemisia sieberi sites in Iran. Journal of Biology, 22(4): 645-660 (In Persian).

9. Assadi, M. 1987. Plants of Arasbaran protected area, NW. Iran (Part I). Iranian Journal of Botany, 3(2): $129-175$.

10. Assadi, M. 1988. Plants of Arasbaran protected area, NW Iran (part II). Iranian Journal of Botany, 4(1): $1-59$.

11. Batisse, M. 1982. The biosphere reserve: A tool for environmental conservation and management. Environmental. Conservation. 9(10): 1-110.

12. Anonymous. 1994. Comprehensive studies of agricultural development and juniper lakes basin. Comprehensive Consulting Engineers Iran. Volume 2 of Forests and Woodlands, $350 \mathrm{pp}$ (In Persian).

13. Boissier, P.E. 1867-1888. Flora Orientalis. vols. 1-5. Genevae et Basileae. H. Georg, Geneva.

14. Davis, P.H. 1966-1984. Flora of Turkey. Vols. 1, 5, 10. Edinburgh University Press, Edinburgh

15. Ejtehadi, H., H. Zare and T. Amini Shakori. 2003. Study and drawing profiles forest cover along the river valley is sweet, Dodangeh Sari, Mazandaran. Iranian Jornal of Biology, 17(4): 346-356 (In Persian).

16. Esmailzadeh, O., H. Asadi, S.M. Hosseini and A. Ahmadi. 2011. Flora, life form and chorological study of Box tree (Buxus hyrcanus Pojark.) sites in Khybus protected forest, Mazandaran. Journal of Plant Biology, 3(8): 27-40 (In Persian).

17. Ghahreman, A. 1979-1992. Colorful flora of Iran. Research Institute of Forests and Rangelands, Tehran (In Persian).

18. Gholami, A., H. Ejtehadi, F. Ghasemzade and J. Ghorshi. 2006. Biodiversity plant species around conservation Lake Bazangan. Iranian Journal of Biology, 19(4): 398-407. (In Persian)

19. Gurgin Karaji, M., P. Karami and H. Marofi. 2014. Introduction to the flora, life forms and chorology of Saral of Kurdistan (Case study sub catchment Farhadabad). Journal of plant researches Iranian Journal of Biology, 26(4): 510- 525 (In Persian).

20. Hamzeh, B. 2000. Some new and noteworthy plant records from Iran. Iranian Journal of Botany, 8(2): 271-277.

21. Hamzeh, B. and A. Jalili. 2002. A new plant and an interesting record from Iran. Iranian Journal of Botany, 9(2): 187-190.

22. Hamzeh, B. and A. Naqinezhad. 2009. Arthraxon P. Beauv. (Gramineae) and Carex caryophyllea (Cyperaceae), new genus and species records from Iran. Iranian Journal of Botany, 15: 68-71.

23. Hamzeh, B., S.R. Safavi, Y. Asri and A. Jalili. 2010. Floristic analysis and a preliminary vegetation description of Arasbaran Biosphere Reserve, NW Iran. Journal of Rostaniha, 11(1): 1-16. (In Persian)

24. Khajeddin, S.J and H. Yeganeh. 2012. The flora, life form and endangered species of karkas hunting prohibited region, Isfahan, Iran. Iranian Journal of Biology, 25(1): 7-20 (In Persian).

25. Mirzadeh Vaghefi, S.S and M. Rajamand. 2008. Life forms and chrotypes of unwanted weedy plants in the main parks of Tehran. Iranian Journal of Forest and Range Protection Research, 6(1): 29-41. (In Persian)

26. Mobayen, S. 1975-1996. Flora of Iran: vascular plants. Tehran University Press, 1-4 (In Persian).

27. Mohammadzadeh, A. 2012. Evaluation of plant species biodiversity in ecological groups using some noun parametric measures in Arasbaran region (Case Study: Ilginechai and Kalybarchai Watershed), M.Sc thesis, Silviculture and Forest Ecology, Behbahan Khatam Alanbia University of Technology, $162 \mathrm{pp}$ (In Persian).

28. Mozafariyan, V and S. Sanandaji. 2009. Saral flora of Kurdistan. Magazine taxonomy and Biosystematics, 2(3): 59-84 (In Persian).

29. Mozaffarian, V. 2003. A dictionary of Iranian plant names. Farhang Moaser Publication, Tehran. (In Persian)

30. Mozaffarian, V. 2005. Plant classification. Amirkabir, Tehran. 1- 2. (In Persian)

31. Naqinezhad, A., S. Gasimov, A. Jalili, J. Sharifi and F. Azimi Motem. 2012. Study on floristic, life form and plant chorology of wetlands in northern and eastern slopes of Sabalan mountains. Taxonomy and Biosystematics, 4(10): 41-52 (In Persian).

32. Naqinezhad, A., S. Mokhtari and M.R. Joharch. 2015. A study on flora, life forms and chorology of plants at border mountains of Arzaneh- Taybad, Khorassan-e Razavi. Journal of plant Researches Iranian Journal of Biology, 28(1): 199-209 (In Persian). 
33. Nicholes, G.E. 1930. Methods in floristic study of vegetation. Ecology, 11: 127-135.

34. Rechinger, K.H. 1963- 2005. Flora Iranica, nos. 1-175. Akademische University Verlasanstalt, Graz. Austia.

35. Pourbabaei, H., H. Manafi and T. Abedi. 2010. The Investigation of woody speciesdiversity in white Georgian oak tree (Quercus petraea subsp. iberica) sites (case study: Choobeh Daragh and Darana, Arasbaran). Iranian Journal of Forest, 2(3): 197-207 (In Persian).

36. Razavi, S.A. 2008. Flora study of life forms and geographical distribution in Kouhmian region (Azadshahr- Golestan province). Journal of Agricultural Science and Natural Resources, 15(3): 98108 (In Persian).

37. Razavi, S and N. Hassan Abbasi, 2010. A Floristic and Chorology Investigation of Oriental Arborvitae in Sourkesh Reserve (Fazel Abad-Golestan Province). Journal of Wood and Forest Science and Technology, 16(2): 83-100 (In Persian).

38. Raunkiaer, C. 1934. The Life Forms of Plants and Statistical Plant Geography, Clarendon Press, Oxford, $132 \mathrm{p}$.

39. Sagheb Talebi, Kh., S. Sajedi and F. Yazdian. 2004. Iran at the Forest Research Institute of Forests and Rangelands Research Forest, 55 pp (In Persian).

40. Salahi Kojoor, E., R. Tamartash and M.R. Tatyan. 2014. The survey of floristic and life form in summer rangeland of Nekaroud basin. Journal of Conservation and Utilization of Natural Resources, 2(1): 93-102.

41. Sokhanvar, F., H. ejtehadi, J. Vaezi, F. memariani, M.R. Joharchi and Z. ranjbar. 2013. Flora, life form and chorology of plants of the Helali protected area in Khorasan-e Razavi province. Taxonomy and Biosystematics, 5(16): 85-101 (In Persian).

42. Taghipour, Sh., M. Hassanzade and S. Hoseini Sarghin. 2011. Flora, life form and chorology of plants of the region of Ala and Rodzard in Khozestan Province. Taxonomy and Biosystematics, 3(9): 15-30 (In Persian).

43. Teimourzade, A., A. Ghorbani and A.H. Kavianpour. 2015. Study on the flora, life forms and chorology of the south eastern of Namin forests (Asi-Gheran, Fandoghloo, Hasani and Bobini), Ardabil province. Journal of Plant Researches. Iranian Journal of Biology, 28(2): 265-275 (In Persian).

44. Zarezade, A., S.M. Mirvakili and A. Mirhoseini. 2006. Flora, life form and chorology of plants species of the Valle Meeriz Damgahan (Yazd Province). Research and Development in Natural resources, 73(1): 129-137.

45. Zohary, M. 1966-1978. Flora Palaestina. Vols 1-3. The Jerusalem Academic Press, Jerusalem.

46. Zohary, M. 1973. Geobotanical Foundations of the Middle East, Fischer Verlag, Stuttgart, Amsterdam, $765 \mathrm{pp}$. 


\title{
Flora, Life form and Chorology of Plants Species of the Arasbaran Biosphere Reserve (Case Study: Kalybarchai and Ilginechai Watershed Basins)
}

\author{
Allahverdy Mohamadzade ${ }^{1}$, Reza Basiri ${ }^{2}$, Mehrangiz Poladian $^{3}$ and Nasim Zeynali ${ }^{4}$ \\ 1- Ph.D Student, Gorgan Agricultural Sciences and Natural Recourses University \\ (Corresponding Author: ecology2020@yahoo.com) \\ 2- Associate, Behbahan Khatam Alanbia University of Technology, Khozestan \\ 3- Graduate Masters, University of Tehran \\ 4- Graduate Masters, Forestry Department, University of Ilam \\ Received: December 28, 2017 Accepted: February 6, 2018
}

\begin{abstract}
s
Arasbaran region is located in the north west of Iran and north of the east Azarbayjan province and most of these forests are seen in four areas of Kalibarchay, Ilgenechay,Hajilerchay and Selenchay. Result showed that 107 species belonge to 74 genera and 36 families of vascular plants are found. which Dicotyledons with 32 families were the richest group. In terms of abundance, Monocotyledons with 4 families and Gymnospermes with 2 families were in the second and third place respectively and one family with one genus and one specie was also from vascular cryptogam. Families of fabaceae with 18 species, Asteraceae with 13 species. Rosaceae with 8 species, Lamiaceae with 6 species and Poaceae with 6 species were as the most important plant families in term of plant species that included a total of $47.63 \%$ of the total species. Life forms of region plants contains of Hemicryptophytes with 42 species Phanerophytes with 26 species, Therophytes with 25 species, Cryptophytes with 11 species and Chamephytes with 3 species, which among them Rosaceae had the greatest number of Phanerophytes, peas greatest number of Therophytes, chicory greatest number of Hemicryptophytes, mint largest number of Chamephytes and grasses has the highest number of Cryptophytes. According to classifications were made on the basis of Rannkier system and calculation were made Hemicryptophytes with having 39.25 percent share of the total number of species constitute the dominant life forms of the region which respectively after them Phanerophytes with $24.29 \%$ and and Therophytes with $23.36 \%$ and Cryptophytes with $10.28 \%$ and Chamephytes with $2.80 \%$ are in the next orders results of determination of corotype plants of region showed that plants with the Geographical distribution of the Irano-Turanian with $34.5 \%$, Euro- Siberian with $30.5 \%$ are the most important group of the Region
\end{abstract}

Keywords: Plant vegetation, Life forms, Geographical distribution, Arasbaran region 